\title{
Antimicrobial susceptibility and virulence factors of enterococci colonizing intestinal tract of Jordanian infants
}

\section{Abstract}

Objective: This study investigated distribution of enterococci colonizing intestinal tract of infants, their putative virulence factors and antimicrobial susceptibility patterns.

Methods: A total of 82/186 (44.1\%) fecal enterococcal isolates were recovered from infants. All enterococci isolates were identified either E. faecalis or E. faecium using culture and PCR.

Results: A significant higher intestinal colonization of enterococci was detected among non-hospitalized compared to hospitalized patients with a percentage of (72 \% vs. $28 \%$ ), respectively. E. faecalis was the predominant species isolated from both groups (75.6\%). It had also significantly higher virulence factors genes than $E$. faecium, while $E$. faecium had higher rates of antimicrobial resistance than $E$. faecalis. There was no significant factor related to intestinal colonization of enterococci with birth weight, gestational age of mother, gender, type of feeding, present of disease or administration of antibiotics to mothers or infants.

Conclusion: This study presents important epidemiological data on the intestinal colonization of enterococci in both hospitalized and nonhospitalized Jordanian infants.

Key word: Fecal enterococci, Infants, Antimicrobial resistance, Virulence factors 


\section{Introduction}

In recent years, enterococci have emerged as important pathogens, particularly in immuoncompromised patients and intensive care units. They cause frequently more infections in patients hospitalized for a long time or who have received multiple antibiotic therapy $[1,2]$. E. faecalis and

E. faecium are the most prevalent species cultured from human infections and are frequently associated with nosocomial infections worldwide $[3,4,5]$.

The emergence of vancomycin-resistance enterococci (VRE) during recent years has seriously affected the treatment and infection control of these bacteria especially in hospitalized patients. $E$ faecium strains are frequently more resistant than E. faecalis to all antibiotics that are effective in the treatment of vancomycin-susceptible enterococci. This new development leaves clinicians treating VRE infections with limited therapeutic options [6-7].

Enterococci are among the first organisms to colonize the intestinal tract of infants. These microorganisms originate mostly from the intestinal and vaginal flora of the mother. They can be also acquired during ingestion of breast milk, formula milk or from environmental sources. An increase in the prevalence of $E$. faecium has been recently observed to occur among neonatal patients in ICUs in many countries, and this shift is likely to be explained in part by the rapid emergence of VRE [6-7]. In addition, it has been suggested that certain virulence factors of enterococci may play a role in intestinal colonization and later infection [8-10].

This study aimed to investigate the important epidemiological features, including antimicrobial susceptibility and virulence factors of common encountered Enterococcus species in the intestine of Jordanian infants.

\section{Material and Methods}

\section{Study design and patients}

The present study included two examined groups; hospitalized and non-hospitalized infants from the Jordan University Hospital, aged from one day to one year. A total of 93 fecal swab specimens were obtained from hospitalized babies admitted to the neonatal intensive care unit (NICU) of the Jordan University Hospital (JUH) for various diseases, from October 2013 through September 2014. At the same period another 93 of fecal specimens obtained from babies attending for routine clinical examination to the outpatient clinic of the Pediatric Department, JUH. A special information sheet was designed to record demographic and clinical data about the patients including infant's name, age, birth weight, gestational age of mother, gender, type of feeding, type of disease if present and administration of antibiotics at time of fecal sampling or two weeks prior to sampling. This study was approved by the Faculty of Graduate Studies, The Jordan University. Permission was also obtained from the Institutional Ethical Review Board (IERB) at the Jordan University Hospital in Amman, Jordan. An approval has been obtained from all parents of the infants which were informed about the nature of the research.

\section{Culture specimen, identification and susceptibility test}

Fecal swab specimens were collected from babies using sterile cotton swabs pre-moistened with $0.9 \%$ saline. All fecal specimens were transferred immediately to the Microbiology Laboratory/ Faculty of Medicine/ Jordan University, and were inoculated directly onto Bile-Esculin agar plates and incubated for $24-48$ hours at $37^{\circ} \mathrm{C}$. Culture plates were examined to detect the presence of gray to black colonies of enterococci. Initial identification of enterococci to genus level was based on Gram-staining properties, 
catalase negative test, and growth in the presence of $6.5 \% \mathrm{NaCl}$. All Enterococcus spp. isolates were stored in Brain Heart Infusion broth with (15\% glycerol) at $-70{ }^{\circ} \mathrm{C}$ for further analysis.

All Enterococcus species isolates were examined for antimicrobial susceptibility using disc diffusion method according to guidelines of Clinical and Laboratory Standards Institute [11]. Vancomycin, linezolid and teicoplanin susceptibility were determined using Etest (BioMerieux, France ) and was interpreted according to the CLSI guidelines. E. fecalis ATCC 29212 was included as a positive control.

\section{PCR Molecular tests}

Multiplex PCR reaction and primers were used for identification of $E$. faecalis, $E$. faecium isolates by detection of genes encoding D-alanine-D-alanine ligases specific for E.fecalis and E. faecium as reported by Kariyama et al. [12]. The glycopeptide resistance genotypes (vanA, vanB, vanC1 and vanC2/ C3) were also determined by use of primers and multiplex PCR as described by Kariyama et al. [12]. Uniplex PCR and primers were used to detect the following virulence factor genes (efaA fs, efaA fm, gel $E$ ) as reported by Cariolato et al. [13], and (ace, esp, cylA genes ) as performed by Creti et. al. [14 ]. E. faecalis ATCC 29212 was used as a positive control for ace, cylA, efaA genes and $E$. faecalis ATCC 51299 was used as a positive control for esp, gel $E$, cylA, ace and efaA.

\section{Statistical analysis}

Data generated from the study was tabulated as Microsoft Excel sheets and Uploaded to Statistical Package for Social Sciences (SPSS version 19). Frequency and percentage were calculated for the categorical data and Pearson's chi-squared test or Fisher's exact test were applied to determine potential factors associated with $E$. faecalis and $E$. faecium and to determine whether there are any statistical differences between groups. The level of significance was set at a $p$ value of $\leq 0.05$ to test the hypothesis of no association

\section{Results}

A total of 186 hospitalized and non-hospitalized infants aged between one day and one year were included in this study. Of these 107 (57.5\%) were males and 79 (42.5\%) were females. A total of 82/196 (44.1\%) isolates of Enterococcus spp were recovered from fecal samples, of these $62 \mathrm{E}$. faecalis (19 inpatients and 43 from outpatients) and $20 E$. faecium (4 from inpatients and 16 from outpatients) isolates were identified (Table 1). Both E. faecalis and $E$. faecium were significantly more found in outpatient infants than in hospitalized infants ( 72 vs. 28\%) as shown in Table 1.

Antimicrobial susceptibility patterns of 20 E. faecium and 62 E. faecalis using disc diffusion test and

Table 1. Enterococcus species detected by PCR from 186 fecal specimens of each 93 hospitalized and non-hospitalized infants*

\begin{tabular}{|c|c|c|c|c|}
\hline Species & Inpatients No. (\%) & Outpatients No. (\%) & Total No. (\%) & P-Value \\
\hline E. faecium & $4(17.4)$ & $16(27.1)$ & $20(24.4)$ & 0.007 \\
\hline E. faecalis & $19(82.6)$ & $43(72.9)$ & $62(75.6)$ & 0.002 \\
\hline Total & $23(28.0)$ & $59(72.0)$ & $82 / 186(44)$ & 0.001 \\
\hline
\end{tabular}

*Including 57\% males and $43 \%$ females; and there was no significant factor related to occurrence of enterococci with birth weight, gestational age of mother, gender, type of feeding, present of disease or administration of antibiotics to mothers or infants. 
Table 2. Antibiotic susceptibility patterns of 62 E. faecalis and 20 E. faecium isolates

\begin{tabular}{|c|c|c|c|c|c|c|}
\hline \multirow[t]{2}{*}{ Antibiotics } & \multicolumn{3}{|c|}{ E. faecalis* } & \multicolumn{3}{|c|}{ E. faecium* } \\
\hline & Susceptible no. (\%) & $\begin{array}{l}\text { MIC90 } \\
(\mu \mathrm{g} / \mathrm{ml})\end{array}$ & $\begin{array}{l}\text { Range } \\
\mu g / m l\end{array}$ & $\begin{array}{c}\text { Susceptible } \\
\text { No.(\%) }\end{array}$ & $\begin{array}{l}\text { MIC90 } \\
(\mu \mathrm{g} / \mathrm{ml})\end{array}$ & $\begin{array}{l}\text { Range } \\
(\mu \mathrm{g} / \mathrm{ml})\end{array}$ \\
\hline Ampicillin & $62(100)$ & - & - & $14(70.0)$ & - & - \\
\hline Chloramphenicol & $24(38.7)$ & - & - & $13(65.0)$ & - & - \\
\hline Gentamicin** & $38(61.3)$ & - & - & $14(70.0)$ & - & - \\
\hline Levofloxacin & $35(56.5)$ & - & - & $4(20.0)$ & - & - \\
\hline Tigecycline & $62(100)$ & & & $20(100)$ & & \\
\hline Teicoplanin & $62(100)$ & 0.44 & $0.016-2$ & $20(100)$ & 0.65 & $0.16-2$ \\
\hline Linezolid & $62(100)$ & 1.48 & $1-2$ & $20(100)$ & 1.63 & $1-2$ \\
\hline Vancomycin & $62(100)$ & 2.4 & $2-4$ & $20(100)$ & 2.7 & $2-4$ \\
\hline
\end{tabular}

*All isolates of $E$. fecalis and $E$. faecium isolates from inpatients showed much similar higher resistant rates in the range to chloramphenicol, levofloxacin gentamicin, respectively ** high level gentamicin $(120 \mu \mathrm{g} / \mathrm{ml})$

E.test are shown in Table 2. E. faecium strains were resistant to ampicillin, chloramphenicol, and levofloxacin with percentages of $30,20,30$, respectively, while $E$. faecalis strains were resistant to the same antibiotics with percentages of 6.5, 25.8, 9.7, respectively. No resistance to vancomycin, teicoplanin and linezolide was detected in both species using Etest.

Overall, E. faecalis has significantly more virulence determinant genes (efaA $f s$, ace, esp, gel $E, c y \mid A$ ) than the $E$. faecium isolates. The virulence gene (efaA $\mathrm{fm}$ ) was detected only in $65 \%$ of $E$. faecium isolates (Table 3).

\section{Discussion}

This study demonstrates that intestines of Jordanian infants aged less than one year admitted to outpatients clinics or neonatal intensive care unit (NICU) at JUH, were frequently colonized with enterococci $(44 \%)$. The highest carriage rate of en- terococci $(72 \%)$ was significantly $(P$-value $=0.001)$ observed among the non-hospitalized infants (aged $>$ one month), while carriage rate in hospitalized infants accounted only for $28 \%$ (Table 1). This

Table 3. Distribution of virulence genes among 62 E. faecalis and $20 \mathrm{E}$. faecium isolates.

\begin{tabular}{|c|c|c|c|}
$\begin{array}{c}\text { Virulence } \\
\text { gene }\end{array}$ & $\begin{array}{c}\text { E. faecalis } \\
\text { No. (\%) }\end{array}$ & $\begin{array}{c}\text { E. faecium } \\
\text { No. (\%) }\end{array}$ & P-Value* \\
\hline efaA fs & $62(100)$ & $10(50)$ & 0.001 \\
\hline ace & $59(95.2)$ & $12(60)$ & 0.001 \\
esp & $59(95.2)$ & $12(60)$ & 0.001 \\
\hline gelE & $50(80.6)$ & $9(45)$ & 0.001 \\
\hline cylA & $46(74.2)$ & $10(50)$ & 0.001 \\
\hline efaA fm & 0 & $13(65)$ & 0.001 \\
\hline
\end{tabular}

* Significant incidence of all virulence genes in E. faecalis compared to $E$. faecium isolates, except efa $A$ fm was significantly associated with $E$. faecium 
result may due to the fact that most hospitalized infants were on antibiotic treatment which has reduced the colonization with enterococci, especially the susceptible strains. The majority of enterococci isolates were E. faecalis (75.6\%) as confirmed by PCR method. The results of this study are generally in agreement with other recently published studies from various countries including Jordan $[6,7,15,16]$.

A previous Jordanian study has reported that $83 \%$ of nosocomial isolates from patients including infants at $J U H$, over a five-year period were $E$. faecalis, followed by $16 \%$ E. faecium using PCR detection method [16].

A recent study from Greece, showed prevalence of various species of enterocooci isolates from fecal samples of 97 healthy infants after their delivery on day, 4, 30, and 90, and the most frequently identified species was $E$. faecalis (54.6\%) followed by $E$. faecium (12\%) [17]. A study from Germany over a 12 -month period, has also found that $23 \%$ of the 274 infants in a neonatal intensive care unit, were mostly colonized with E. faecalis [18]. The reason for different distribution rates of enterococci species recovered from fecal specimens of infants and others in various countries may be related to the types and amount of antibiotics usage in these countries which are known to contribute rapidly for change of the gut microbiota [3].

The present study demonstrates that most E.faeacalis and E. faecium isolates were highly susceptible to most tested antibiotics, and E. faecalis isolates showed more high rates of susceptibility than E. faecium to ampicillin, chloramphenicol, gentamicin and levofloxacin in the range (93.5-65.5\%). In addition, all $E$. fecalis and $E$. faecium isolates from inpatients showed higher resistant rats to chloramphenicol than those isolates from outpatients (Table 3). Additionally, similar results has been re- ported by a previous Jordanian study where all $E$. faecalis isolated from patients with dental diseases and healthy controls were $100 \%$ susceptible to ampicillin, ciprofloxacin, chloramphenicol, vancomycin, and teicoplanin, but to less extent to erythromycin $62.5 \%$ [19]. However, numerous recent studies from various continents have reported an increasing occurrence of infection due to multidrug resistance of E. faecalis and E. faecium, and some of these studies documented increasing incidence of vancomycinresistant in Enterococcus spp. isolated from serious human infections including infants $[4,7,9,20]$.

This study shows that $E$. faecalis isolates carried statistically significant more virulence determinants than the E. faecium isolates (Table 3 ). The present study has also demonstrated that both Enterococcus species isolates from hospitalized infants harbored more virulence genes than isolates from non-hospitalized. This result is different from other published study which has indicated that intestinal enterococci isolates from outpatients carry slightly more virulence genes than isolates from hospitalized patients [10].

However, The overall results of this study are comparable to the results represented by other worldwide studies which showed that E. faecalis carried higher rates and more multiple virulence determinates as compared with E. faecium [21-24]. Additionally, this study has detected high occurrence rates of most important putative virulence factors in enterococci isolates from infants, including collagen-binding protein (Ace). However, it has been shown that enterococcal virulence determinates are still considered a complex issue in causing infection because the essential factors for their pathogenic potential have not yet been well described [22]

In conclusion, this study shows important epidemiological features of fecal enterococci isolates 
from Jordanian infants, especially their susceptibility to frequently used antimicrobials and association with potential virulence determinates.

There is no conflicts of interest related to this study.

\section{Acknowledgment}

This study has been financially supported by The Dean of Research, The Jordan University, and The Jordanian Research Fund, Amman, Jordan.

\section{References}

1. Chakraborty A, Pal KN, Sarkar S, Gupta MS. Antibiotic resistance pattern of Enterococci isolates from nosocomial infections in a tertiary care hospital in Eastern. India J Nat Sci Biol Med 2015; 6(2): 394-397.

2. Furtado I, Xavier PCN, Tavares LVM, Alves F, Martins SF, et al. Enterococcus faecium and Enterococcus faecalis in blood of newborns with suspected nosocomial infection. Rev Inst Med Trop Sao Paulo 2014; 56(1):77-80

3. Arias C A, Murray BE. 2012. The rise of the Enterococcus: beyond vancomycin resistance. Nat Rev Microbiol 2012; 16:26678.

4. Pan SC, Wang TJ, Chen CY, Chang YY, Chen LM, et al. Incidence of and Risk Factors for Infection or Colonization of Vancomycin-Resistant Enterococci in Patients in the Intensive Care Unit. PLoS. ONE 2012; 7(10): e47297.

5. Daniel DS, M.Lee S, Dykes AG, Rahman S. Public Health Risks of Multiple-Drug-Resistant Enterococcus spp. in Southeast Asia. Appl Environ Microbiol 2015; 81(18): 6090-7.

6. Shehabi AA., Badran FE, Alnasra AN. The emergence of antimicrobial resistance in enterococci isolates from infants: A review study. IAJAA 2014;4: (3)5.

7. Werner G, Coque TM, Franz CM, Grohmann E, Hegstad K, et al. 2013 Antibiotic resistant enterococci-tales of a drug resistance gene trafficker. Int JMed Microbiol 2013;303: 360-79.
8. Dahlen G, Blomqvist S, Almstahl A, Carlen A. Virulence factors and antibiotic susceptibility in enterococci isolated from oral mucosal and deep infections. J Microbiol 2012; 4:10.

9. Shankar N, Lockatell CV,.Baghdayan AS, C. Drachenberg C, Gilmore MS, et al. Role of Enterococcus faecalis surface protein Esp in the pathogenesis of ascending urinary tract infection, Infect Immuol 2001; 69:4366-4372.

10. Lempiäinen $H$, Kinnunen $K$, Mertanen $A$, von Wright $A$. Occurrence of virulence factors among human intestinal enterococcal isolates. Lett Appl Microbiol 2005; 41 (4): 341-4.

11. CLSI Performance Standards for Antimicrobial Susceptibility Testing, Twentieth Informational Supplement (2013), Wayne, PA: Clinical and Laboratory Standards Institute. CLSI document M100-S20.

12. Kariyama R, Mitsuhata R, Chow JWR, Clewell DB, Kumon H. 2002. Simple and reliable multiplex PCR assay for surveillance isolates of vancomycin-resistant enterococci. J Clin Microbiol 2002; 38: 3092-5.

13. Cariolato $D$, Andrighetto $C$, Lombardi A. Occurrence of virulence factors and antibiotic resistances in Enterococcus faecalis and Enterococcus faecium collected from dairy and human samples in North Italy. Food Control 2008; 19: 886-892.

14. Creti R, Imperi M, Bertuccini L, Fabretti F, Orefici G, et al. 2004. Survey for virulence determinants among Enterococcus faecalis isolated from different sources. J Med Microbiol 2004; 53: 1320.

15. Jia W, Li G, Wang W. Prevalence and Antimicrobial Resistance of Enterococcus Species: A Hospital-Based Study in China. Int J Environ Res Pub Health 2014;11: 3424-3442.

16. Mahafzah A, Abu-Khader I, Bakri F. Characterization of Enterococci Causing Nosocomial Infections at the Jordan University Hospital Over a Five-year Period. Jord Med J 2008; 42 (4): 229-237.

17. Kirtzalidou El, Mitsou EK, Pramateftak P, Kyriacou A. 2012. Screening Fecal Enterococci from Greek Healthy Infants for Susceptibility to Antimicrobial Agents. Microbial Drug Resist 2012; 18(6):578-585.

18. Hufnagel M, Liese $C$, Loescher $C$, Kunze $M$, Proempeler $H$, et al. Enterococcal colonization of infants in a neonatal intensive care unit: associated predictors, risk factors and seasonal patterns. BMC Infect Dis 2007;7:107.

19. Salah R, Dar-Odeh N, Abu Hammad O, Shehabi AA. Prevalence of putative virulence factors and antimicrobial susceptibility of E. faecalis isolates from patients with dental Diseases. BMC Oral Health 2008; 8:17. 
20. Ochoa SA, Escalona G, Cruz-Córdova A, Dávila LB, Saldaña Z, et al. Molecular analysis and distribution of multidrug-resistant Enterococcus faecium isolates belonging to clonal complex 17 in a tertiary care center in Mexico City. BMC Microbiol 2013; 11:13-291.

21. Fisher $K$, Phillips $C$. The ecology, epidemiology and virulence of Enterococcus, Microbiology 2009; 155: 1749-57.

22. Solheim M, Aakra, Snipen GL, A.D. Brede DA, Nes FI. Comparative genomics of Enterococcus faecalis from healthy Norwegian infants. BMC Genomics 2009; 10:194-205.

23. Hasani A, Sharifi Y, Ghotaslou R, Naghili B A, Aghazadeh M. et al. Molecular screening of virulence genes in high-level gentamicin-resistant Enterococcus faecalis and Enterococcus faecium isolated from clinical specimens in Northwest Iran. Ind J Med Microbiol 2012; 30(2):175-81.

24. Hallgren A, Claesson C, Saeedi B, Monstein HJ, Hanberger $H$, et al. Molecular detection of aggregation substance, enterococcal surface protein, and cytolysin genes and invitro adhesion to urinary catheters of $\mathrm{E}$. faecalis and $\mathrm{E}$. faecium of clinical origin. Int J Med Microbiol 2009; 299: 323-332.

\section{Comment on this article:}

\section{(f) B in $8+\mathbf{S} P$}

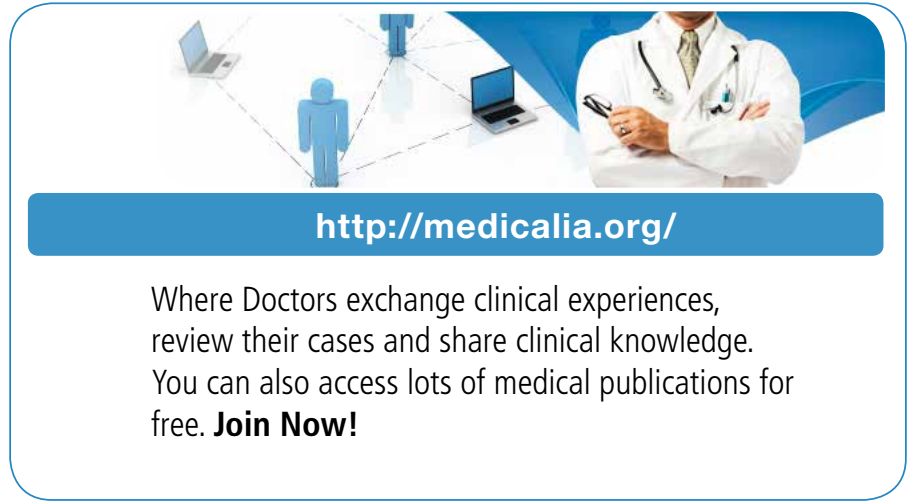

\section{Publish with iMedPub}

http://www.imed.pub

The Journal is an open access peer-reviewed journal that publishes scientific papers about all aspects of antimicrobials. The journal will publish original research articles, reviews, brief reports and case reports dealing with basic and clinical antibacterial agents, antiviral, antiprotozoals, antituberculuous, antifungal and antihelminthes agents.

All manuscripts must be prepared in English, and are subject to a rigorous and fair peer-review process. Accepted papers will immediately appear online.

The journal aims to advance the knowledge, attitude and the research of chemotherapy in the Arabic world in cooperation with international, national scientific and public societies as well as research centers with similar aims and objectives. 